\title{
Interaction of Lysozyme Protein with Different Sized Silica Nanoparticles and Their Resultant Structures
}

\author{
Indresh Yadav ${ }^{1, *}$, V. K. Aswal ${ }^{1}$ and J. Kohlbrecher ${ }^{2}$

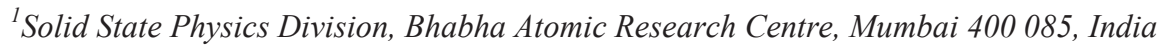 \\ ${ }^{2}$ Laboratory for Neutron Scattering, Paul Scherrer Institut, CH-5232 PSI Villigen, Switzerland \\ "E-mail: iykumarindresh288@gmail.com
}

\begin{abstract}
The interaction of model protein-lysozyme with three different sized anionic silica nanoparticles has been studied by UV-vis spectroscopy, dynamic light scattering (DLS) and small-angle neutron scattering (SANS). The surface area and curvature of the nanoparticles change with size, which significantly influence their interaction with protein. The lysozyme adsorbs on the surface of the nanoparticles due to electrostatic attraction and leads to the phase transformation from one phase (clear) to two-phase (turbid) of the nanoparticle-protein system. The dominance of lysozyme induced short-range attraction over long-range electrostatic repulsion between nanoparticles is responsible for phase transformation and modeled by the two-Yukawa potential. The magnitude of the attractive interaction increases with the size of the nanoparticles as a result the phase transformation commences relatively at lower concentration of lysozyme. The structure of the nanoparticle-protein system in two-phase is characterized by the diffusion limited aggregate type of mass fractal morphology.
\end{abstract}

Keywords: Colloids, nanoparticle-protein interaction, small-angle neutron scattering PACS: 82.70.Dd, 61.46.-w, 61.05.fg

\section{INTRODUCTION}

Colloidal dispersions of nanoparticles and proteins show rich phase behavior and have wide applications in drug delivery, catalysis, biological imaging, biosensors, novel functional materials, to name a few [1]. The characteristics of nanoparticles and proteins (e.g. size, shape, charge, and hydrophilicity or hydrophobicity) and the forces (e.g., electrostatic, hydrogen bonding, and steric forces) between them, govern their phase behavior, which can be modulated by ionic strength, $\mathrm{pH}$, temperature and concentration, etc. The size of the nanoparticles plays vital role in the nanoparticle-protein interactions [1]. Tuning the size of the nanoparticles not only change the surface area but also the curvature of the nanoparticles for protein interaction. In the case of protein adsorbing on nanoparticles, the competition of decreasing curvature (favoring adsorption) and decreasing overall surface area (disfavoring adsorption) with increasing nanoparticle size controls the adsorption behavior of protein on the nanoparticles [2]. This in turn is also important for the colloidal stability of the nanoparticles and their resultant structure. In the case of charged nanoparticles, electrostatic interactions dictate the phase behavior of the nanoparticle-protein system and has been found that protein adsorb on the oppositely charged surface, but not otherwise [3-4]. The adsorption of oppositely charged protein could lead to the protein mediated aggregation of the nanoparticles where protein serves as a glue to bind the nanoparticles. Herein, we have studied the effect of the size of the nanoparticles on their interaction with protein. The adsorption isotherm as well as interactions and resultant structure in these systems have been measured by a combination of spectroscopy (UV-Vis) and scattering (DLS and SANS)

techniques.

\section{EXPERIMENT}

Colloidal suspensions of three different sized spherical silica nanoparticles (Ludox SM30, HS40, and TM40) and lyophilized hen egg protein lysozyme were purchased from Sigma-Aldrich. Different compositions of nanoparticle-protein samples were prepared in $20 \mathrm{mM}$ phosphate buffer at $p \mathrm{H} 7$ keeping concentration of silica nanoparticles (1 wt \%) fixed and varying the concentration of proteins in the range 
0 to 5 wt $\%$. Small-angle neutron scattering experiments were performed on the SANS-I instrument at the Swiss spallation neutron source, SINQ, Paul Scherrer Institut, Switzerland. The mean wavelength $(\lambda)$ of the incident neutron beam was $6 \AA$ with the wavelength resolution of approximately $10 \%$. A large $\left(96 \times 96 \mathrm{~cm}^{2}\right) \mathrm{He}-3,2 \mathrm{D}$ detector is used to collect the scattered neutrons. The data were corrected and normalized to absolute scale using standard procedure. The temperature was kept constant at $30^{\circ} \mathrm{C}$ during all the measurements.

\section{SANS ANALYSIS}

In SANS, coherent differential scattering crosssection per unit volume $(d \Sigma / d \Omega)$ is measured as a function of scattering vector $Q$, and for a system of interacting monodisperse particles it can be written as [3].

$$
\frac{d \Sigma}{d \Omega}(Q)=n V^{2}\left(\rho_{p}-\rho_{s}\right)^{2} P(Q) S(Q)+B
$$

where $n$ is the number density and $V$ is the particle volume. $\rho_{\mathrm{p}}$ and $\rho_{\mathrm{s}}$ are scattering length densities of particles and solvent, respectively. $P(Q)$ is intraparticle structure factor, a measure of spatial correlation of scattering within the particle, and gives information about the shape and size of the particle. $S(Q)$ is interparticle structure factor, it describes the correlation of scattering from different particles, and hence contains the information about the interactions between particles and their spatial arrangements. $B$ is a constant term representing incoherent background.

\section{RESULTS AND DISCUSSION}

The individual nanoparticles and lysozyme protein have been characterized by SANS. The mean radii of SM30, HS40, and TM40 are found to be 4.2, 8.8 , and $13.8 \mathrm{~nm}$, respectively. On the other hand, lysozyme has a prolate ellipsoid shape with semimajor axis $2.40 \mathrm{~nm}$ and semiminor axis $1.35 \mathrm{~nm}$ [3-5]. The significant difference in size of nanoparticles and protein allows enough number of proteins to interact with the nanoparticles. Further, at $\mathrm{pH} 7$ strong Coulombic interactions could be anticipated between the negatively charged silica nanoparticles and positively charged lysozyme. The adsorption isotherm of lysozyme on silica nanoparticles as obtained by UV-vis spectroscopy is presented in Figure 1. It shows the amount of adsorbed lysozyme (wt \%) versus total lysozyme on 1 wt \% three different sized silica nanoparticles. Free proteins, if any, from that of adsorbed on the surface of the nanoparticles were separated by centrifugation. For lysozyme, the absorption spectrum shows a peak at about $280 \mathrm{~nm}$.
The absorption of incident light is proportional to the concentration of free protein. Thereby, by calculating the ratio of absorbance of supernatants to that of corresponding pure protein solution we have calculated the concentration of free protein. The adsorption curve shows an exponential behavior irrespective of the size of the nanoparticles. At constant volume fraction, smaller sized nanoparticles will provide overall larger surface area for the adsorption of proteins. The saturation values (in wt \%) are found to be $0.67,0.50$ and 0.31 for SM30, HS40 and TM40 nanoparticles, respectively. The number of adsorbed lysozyme per nanoparticles are also calculated and found to be 19, 130 and 310 for SM30, HS40 and TM40 nanoparticles, respectively. It is also observed that the adsorbed lysozyme is not scaled by the surface area due to curvature effect.

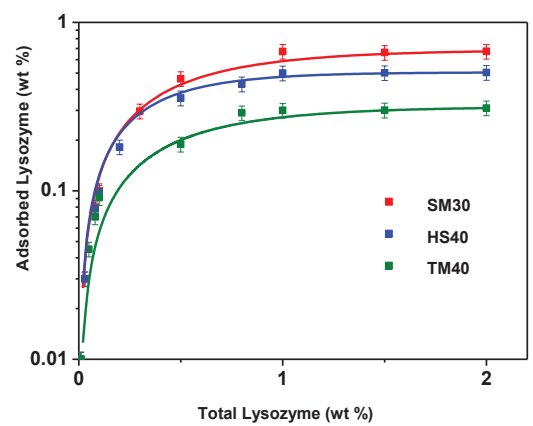

Figure 1. Adsorption isotherm of lysozyme on $1 \mathrm{wt} \%$ silica nanoparticles. Lysozyme shows an exponential behavior irrespective of the nanoparticles size.

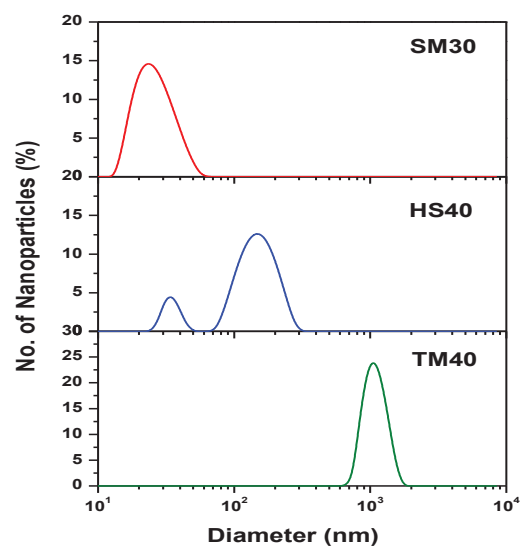

Figure 2. Particles size distribution of three different sized nanoparticles in presence of $0.04 \mathrm{wt} \%$ lysozyme.

Figure 2 shows the size distribution of three different sized silica nanoparticles in presence of 0.04 wt \% lysozyme as obtained by DLS. It is clear that same amount of lysozyme give rises to different structural evolution for different sized nanoparticles. Lysozyme induces the aggregation of the nanoparticles 
Table 1. Fitted parameters of SANS data of $1 \mathrm{wt} \%$ silica nanoparticles (SM30, HS40, and TM40) + C wt \% Lysozyme. The parameters of repulsive interaction of two-Yukawa potential $\left(K_{2}=9.0\right.$ and $\left.Z_{2}=7.0\right)$ were kept fixed.

\begin{tabular}{|c|c|c|c|c|}
\hline \multirow[b]{2}{*}{ Nanoparticle System } & \multicolumn{2}{|c|}{0.005 wt \% Lysozyme } & \multicolumn{2}{|c|}{0.5 wt \% Lysozyme } \\
\hline & $\overline{K_{1}}$ & $\bar{Z}$ & $\begin{array}{c}\text { Fractal dimension } \\
D\end{array}$ & $\begin{array}{c}\text { Building block radius } \\
R_{\mathrm{b}}(\mathrm{nm})\end{array}$ \\
\hline SM30 & - & - & 2.2 & 5.5 \\
\hline HS40 & 14 & 8 & 2.5 & 9.0 \\
\hline TM40 & 52 & 8 & 2.7 & 14.8 \\
\hline
\end{tabular}

By mediating between them. For fixed volume fraction, number density of SM30 is smaller than HS40 and TM40. Thus the same concentration of lysozyme induces the higher degree of aggregation in bigger sized nanoparticles. In case of SM30 scattering of light is mainly governed by monomers as a result it shows the single peak in size distribution. HS40 shows two peaks in size distribution i.e. larger contribution coming from aggregated nanoparticles whereas for TM40 scattering is completely dominated by aggregates and the system has completely been transformed to two-phase system.
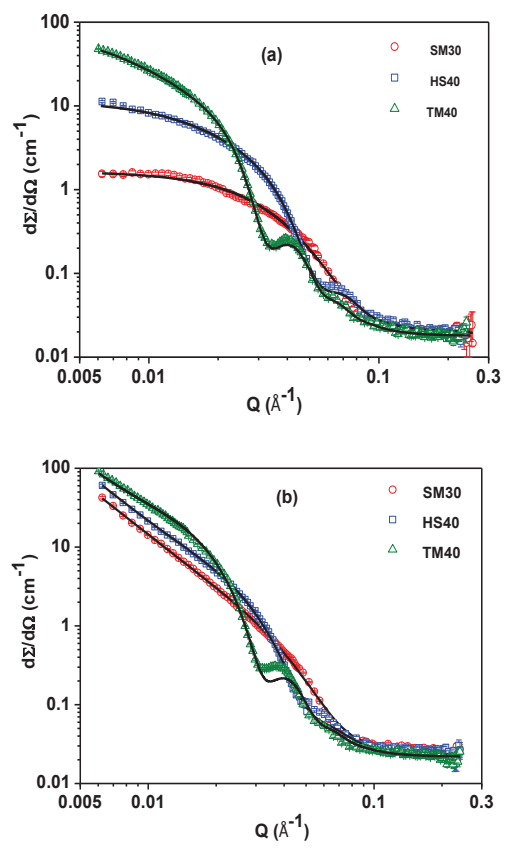

Figure 3. SANS data of $1 \mathrm{wt} \%$ silica nanoparticles (SM30, HS40 and TM40) in presence of (a) $0.005 \mathrm{wt} \%$ and (b) 0.5 wt \% lysozyme.

Figure 3 shows the SANS data of silica nanoparticles at two different concentration of lysozyme. The data of Fig 3(a) has been modeled by the two-Yukawa potential under mean spherical approximation. The potential comprises four fitting parameters $\left(K_{1}, K_{2}, Z_{1}, Z_{2}\right)$, where $K_{1}$ and $Z_{1}$ are the fitting parameters of the attractive potential to give the strength (proportional to $K_{1}$ ) and range (proportional to $1 / Z_{1}$ ), respectively [3]. $K_{2}$ and $Z_{2}$ are the fitting parameters of the repulsive potential which provide the strength (related to effective charge) and range (related to ionic strength), respectively. $K_{2}$ and $Z_{2}$ were kept fixed and found to be almost similar for all three different sized nanoparticles. Whereas, $K_{1}$ and $Z_{1}$ are treated as fitting parameters whose values are given in table 1 . In case of SM30 at 0.005 wt \% lysozyme data were fitted merely by form factor of nanoparticles. On the other hand, at $0.5 \mathrm{wt} \%$ [Fig. 3(b)] data are found to be linear at low $\mathrm{Q}$ which is signature of aggregates. The aggregates of nanoparticles-protein system are found to have mass fractal morphology. The geometrical packing will be more for higher value of size ratio of nanoparticles and protein. Thus the fractal dimension increases with the nanoparticles size.

\section{CONCLUSION}

The size-dependent interaction of silica nanoparticles with oppositely charged lysozyme protein has been studied. The adsorption isotherm of protein shows exponential behavior for all the different sizes of nanoparticles and the saturation value of adsorption depends on the total surface area of nanoparticles. The adsorption leads to proteinmediated aggregation of nanoparticle and is enhanced with the increase in the nanoparticle size. The attractive interaction is short-range, whose magnitude is determined by the amount of adsorption on nanoparticles and can be varied by the change in nanoparticle size and/or number density of nanoparticles.

\section{REFERENCES}

1. I. Lynch and K.A. Dawson, Nano Today 3, 40 (2008).

2. A. A. Vertegel, R. W. Siegel, and J. S. Dordick, Langmuir, 20, 6800 (2004).

3. I. Yadav, S. Kumar, V. K. Aswal, and J. Kohlbrecher, Phys. Rev. E 89, 032304 (2014).

4. I. Yadav, V. K. Aswal, and J. Kohlbrecher, Phys. Rev. E 91, 052306 (2015).

5. S. Kumar, V.K. Aswal and P. Callow, Langmuir 30, 1588 (2014).

6. Y. Liu, W.-R. Chen, and S.-H. Chen, J. Chem. Phys. 122, 044507 (2005). 